\title{
BSC E A ESTRUTURA DE ATRIBUTOS DA CONTABILIDADE GERENCIAL: UMA ANÁLISE NO AMBIENTE BRASILEIRO
}

\author{
THE BSC AND THE ATTRIBUTES STRUCTURE OF MANAGEMENT ACCOUNTING: AN ANALYSIS WITHIN THE BRAZILIAN ENVIRONMENT
}

BSC Y LA ESTRUCTURA DE ATRIBUTOS DE LA CONTABILIDAD GERENCIAL: UN ANÁLISIS EN EL AMBIENTE BRASILEÑO

\section{RESUMO}

Este artigo tem por objetivo investigar se existe associação entre a estrutura de atributos da contabilidade gerencial e a existência do balanced scorecard (BSC) em empresas brasileiras. A pesquisa utilizou a estrutura de atributos da contabilidade gerencial desenvolvida por Chenhall e Morris (1986) e aperfeiçoada por Moores e Yuen (2001). Por meio dessa associação, buscou-se identificar as características das empresas que implantaram ou estão em fase de implementação do BSC e analisar as possíveis causas para sua pouca utilização. Foi possível identificar relações significativas entre a implantação do BSC e os atributos da contabilidade gerencial em três dos quatro grupos de empresas discriminados pelas variáveis de questionamento. A utilidade do conhecimento revelado reside no potencial de orientação aos gestores quanto aos requisitos e condições estruturais para uso do BSC, diminuindo o risco de insucesso e facilitando a implementação.

PALAVRAS-CHAVE Balanced scorecard, contabilidade gerencial, controle gerencial, desempenho empresarial, atributos da contabilidade.

Fábio Frezatti frezatti@usp.br

Professor da Faculdade de Economia, Administração e Contabilidade, Universidade de São Paulo - São Paulo - SP, Brasil

Tania Regina Sordi Relvas taniarsr@usp.br

Professora da Faculdade de Economia, Administração e Contabilidade, Universidade de São Paulo - São Paulo - SP, Brasil

Emanuel Junqueira emanueljunqueira@ccje.ufes.br

Professor do Departamento de Ciências Contábeis, Universidade Federal do Espírito Santo - Vitória - ES, Brasil

Recebido em 10.04.2008. Aprovado em 21.03.2010

Avaliado pelo sistema double blind review. Editor Científico: Ricardo R. Rochman

\begin{abstract}
This paper aims to research the association between the management accounting attribute structure and the existence of the balanced scorecard (BSC) in the Brazilian companies. The research used the management accounting attribute structure developed by Chenhall and Morris (1986) and improved by Moores and Yuen (2001). Considering this association, the research tried to identify companies' characteristics of the ones that implemented or are in the processing of BSC implementation and analysed the possible reasons of its rare usage. It was possible identify significant relationship between BSC implementations and management accounting attributes profile in three of the four defined groups. The knowledge of this research is useful due to the potential orientation that it could provide for management in the requirements and structural conditions for BSC usage reducing the risk of failure and improving the implementation process.
\end{abstract}

KEYWORDS Balanced scorecard, management accounting, management control, business performance, accounting attributes.

RESUMEN Este artículo tiene el objetivo investigar si existe asociación entre la estructura de atributos de la contabilidad gerencial y la existencia del balanced scorecard (BSC) en empresas brasileñas. La investigación utilizó la estructura de atributos de la contabilidad gerencial desarrollada por Chenhall y Morris (1986) y perfeccionada por Moores y Yuen (2001). Por medio de esa asociación, se buscó identificar las características de las empresas que han implementado o se encuentran en el curso de la implementación del BSC, y analizar las posibles causas para su poca utilización. Fue posible identificar relaciones significativas entre la implantación del BSC y los atributos de la contabilidad gerencial en tres de los cuatro grupos de empresas discriminados por las variables de cuestionamiento. La utilidad del conocimiento revelado reside en el potencial de orientación a los gestores respecto a los requisitos y condiciones estructurales para uso del BSC, disminuyendo el riesgo de fracaso y facilitando la implementación.

PALABRAS CLAVE Balanced scorecard, contabilidad gerencial, control gerencial, desempeño empresarial, atributos de la contabilidad. 


\section{INTRODUÇÃO}

O balanced scorecard, doravante referenciado como BSC, é um instrumento de gestão que faz parte dos elementos de artefatos da contabilidade gerencial (CHENHALL, 2003; ITTNER; LARCKER; MEYER, 2003), e que desde a sua divulgação tem passado por vários estágios de desenvolvimento. Considerado um artefato (WICKRAMASINGHE e ALAWATTAGE, 2007), o BSC passa a fazer parte do elenco de artefatos da contabilidade gerencial que uma organização pode escolher implementar e, caso seja aceito pelos gestores, torna-se uma das formas de controle gerencial (BURNS; SCAPENS, 2000).

A perspectiva de transformar a estratégia em ação, operacionalizando as decisões de longo prazo, se constitui em algo relevante dentro do universo empresarial. Diversos autores enfatizam a relevância do alinhamento entre a estratégia e o desempenho (KAPLAN; NORTON, 1992, 1996; NEELY; AUSTIN, 2000; TONCHIA, 2000). O foco do BSC, como proposto por Kaplan e Norton (1992), é justamente estabelecer uma relação entre a eficácia na gestão e as operações por meio do alinhamento entre a estratégia de negócio e o desempenho operacional, utilizando uma composição balanceada de medidas, metas, objetivos e requisitos para essa finalidade.

Scapens (1994) menciona que os artefatos mais recentes são relativamente pouco utilizados e recomenda que as pesquisas sejam mais focadas no entendimento de como as entidades os implementam. Algumas pesquisas indicam que o BSC representa uma evolução em relação a modelos conceituais anteriores, tais como a administração por objetivos (RODRIGUES, 2001) e o tableau de bord (EPSTEIN; MANZONI, 1998; ROSA, 2000). Entretanto, o arcabouço teórico desse artefato ainda não está consolidado e o seu desenvolvimento ocorre em várias partes do mundo, conduzindo a diferentes perspectivas e formas de utilização, que podem ser relacionadas com questões culturais e macroeconômicas, entre outras (AX; BJORNENAK, 2005; FERNANDES e outros, 2006; DAVIS; ALBRIGHT, 2004; SPECKBACKER e outros, 2003). Apesar da pouca utilização destacada por Scapens (1994), pesquisas recentes, como a realizada por Chenhall (2003), apontam para um crescimento na utilização do BSC ou instrumentos similares de avaliação com múltiplos indicadores e perspectivas.

Chenhall (2003, p. 132) contribui para o entendimento do crescimento da utilização do BSC ao destacar que a estrutura do sistema de controle gerencial das organizações está relacionada com a utilidade dos artefatos para os gestores. Ou seja, se determinado artefato, como, por exemplo, o BSC, for considerado útil na gestão dos negócios, é provável que seja utilizado na busca de melhores decisões visando às metas organizacionais.

Isso permite inferir que a qualidade das decisões dos gestores pode ser significativamente influenciada dependendo dos artefatos que integram o sistema de controle gerencial da empresa, o que, direta ou indiretamente, afeta o desenvolvimento e a competitividade das empresas e do mercado como um todo.

No Brasil, apesar do mercado robusto em termos do Produto Interno Bruto (PIB) e do grande número de empresas, os estudos sobre a implementação e utilização do BSC (SOARES JÚNIOR, 2003) indicam que a utilização desse artefato é ainda menor do que em outros países. Em uma pesquisa de campo desenvolvida em 2002 (FREZATTI, 2005), que investigou empresas de médio e grande porte, constatou-se que, em uma amostra probabilística de 119 empresas, apenas 13\% responderam que utilizavam o BSC. Embora tais resultados estejam consistentes com o relato de Scapens (1994), eles são modestos em relação ao que tem sido percebido em vários outros países (KALD; NILSSON, 2000).

Como possíveis explicações para isso, pode-se citar o desconhecimento dos gestores sobre o artefato e/ou seu potencial de benefícios, bem como dos pré-requisitos e condições estruturais para sua implementação e uso. Nesse sentido, o nível de conhecimento dos gestores da organização sobre o artefato passa a ser relevante no entendimento do tema, bem como a investigação se as condições estruturais para implantá-lo estão sendo atendidas. Na presente pesquisa, tais condições foram limitadas à estrutura de atributos da contabilidade gerencial que estavam disponíveis nas empresas pesquisadas, e procurou-se relacioná-las com as demandas necessárias para a implantação do BSC.

Diante desse contexto, o objetivo geral do estudo é investigar se existe alguma associação entre a estrutura de atributos da contabilidade gerencial e a existência do balanced scorecard (BSC). Portanto, a questão de pesquisa que norteia este trabalho é: "Existe alguma relação entre a estrutura de atributos da contabilidade gerencial e a utilização do BSC nas empresas de médio e grande porte no Brasil?".

No desenvolvimento da pesquisa, foram utilizadas variáveis de questionamento para discriminar os grupos de empresas em termos do conhecimento e da utilização do BSC, mas não foram levantados dados sobre a 'finalidade de implantação' ou a 'intensidade de uso' do BSC. A contribuição para a literatura está no entendimento de como os pré-requisitos de um artefato, no caso o BSC, podem 
influenciar a sua implantação ao verificar se as empresas que efetivamente utilizam o BSC possuem ou não esses prérequisitos, o que no último caso indicaria um alto risco para o sucesso desse instrumento pela falta de sustentação em termos de estrutura e informação necessárias.

\section{REVISÃO DA LITERATURA}

A revisão da literatura foi baseada nos seguintes temas: utilidade dos artefatos da contabilidade gerencial, balanced scorecard e dificuldades de implementação, e atributos da contabilidade gerencial.

\section{A utilidade dos artefatos do sistema de informações da contabilidade gerencial}

Chenhall e Morris (1986, p. 17) enfatizam a 'utilidade' das características da informação fornecida pelo SICG (Sistema de Informações da Contabilidade Gerencial) e, com base em estudos relativos à utilidade percebida da informação, afirmam que os indivíduos têm suficiente autonomia no processo de decisão para entender a importância da informação em suas atividades.

Segundo Chenhall (2003, p. 132), muitos estudos têm confirmado as características genéricas de escopo 'amplo', 'tempestividade', 'agregação' e 'integração' do Sistema de Controle Gerencial, sendo que os resultados devem ser separados dentro de questões relacionadas ao uso ou à utilidade do sistema e aos resultados comportamentais e organizacionais decorrentes.

Chong (1996, p. 416) constatou que "[...] ligar 'utilidade percebida' das características da informação produzida pelo SICG ao desempenho gerencial é problemático devido à 'extensão do uso' provocar impactos sobre o desempenho gerencial". Chenhall (2003, p. 135) também chama a atenção para as questões de 'uso' e 'utilidade' dos sistemas de controle gerencial. Isso é relevante na medida em que existem muitos artefatos que as pessoas utilizam, mas não por escolha pessoal ou convicção de sua utilidade, ou seja, artefatos que as pessoas são forçadas a usar na tomada de decisões, tais como orçamento ou análise do fluxo de caixa descontado, mesmo quando os consideram como de pouca utilidade. Dessa forma, ainda que determinados artefatos sejam percebidos como pouco úteis, com baixa taxa de satisfação ou de poucos benefícios, um alto desempenho organizacional pode ser alcançado devido à obtenção das informações desejadas de fontes, formais ou informais.

Woulters e Veerdaasdonk (2002) revelam que alguns estudos têm demonstrado que os gestores desejam in- formações tempestivas, incorporando dados não contábeis e externos diante da presença de incerteza ou de interdependências entre unidades organizacionais ou tarefas, e que, em certas situações, os gestores classificam a informação contábil que é fornecida como sendo de pouca 'utilidade' para a tomada de decisão. Por um lado, podemos questionar até que ponto os gestores estão atentos para o potencial de benefícios das informações contábeis, mas, de outro, podemos constatar que o BSC, enquanto artefato, incorpora tanto a informação financeira quanto a não financeira, o que potencialmente atende às demandas dos gestores e amplia o número de usuários das informações.

Pierce e O'Dea (2003, p. 269) analisaram a percepção dos gestores em relação às exigências que fazem em termos de informações e à percepção que têm da eficácia para atender a tais exigências, e constataram "[...] diferenças substantivas entre a percepção dos contadores gerenciais e dos gestores relativas à natureza específica da informação considerada essencial pelos gestores e a qualidade da informação fornecida a eles por meio do sistema de contabilidade gerencial [...]", indicando também que existe um sensível gap entre o que a contabilidade gerencial proporciona aos usuários e o que estes acreditam ser útil à gestão. Nesse raciocínio, Abernethy e Vagnoni (2004) sugerem que o uso da informação contábil é dependente da percepção gerencial relativa ao desenho associado com o sistema. Assim, à medida que os gestores percebem que a informação é relevante e acurada para a tomada de decisões, passam a usá-la.

\section{Balanced scorecard e dificuldades de implementação}

O BSC, quando introduzido por Robert S. Kaplan e David P. Norton, em 1992, tinha como foco a comunicação do desempenho da organização por meio de indicadores (financeiros e não financeiros). Posteriormente, foi redirecionado para avaliar o desempenho por meio de uma rede de medidas e indicadores que refletissem a estratégia de negócio da organização em todos os níveis. As medidas são organizadas em quatro perspectivas: financeira, dos clientes, dos processos internos, e aprendizado e crescimento. Cada perspectiva envolve um conjunto específico de indicadores ajustados em relação às características e demandas específicas da organização com foco na visão e estratégia.

Paranjape e outros (2006) constataram que o insucesso na implantação do BSC nas organizações está relacionado com um ou mais dos seguintes fatores: (a) seleção de medidas impróprias ou excessivas; (b) implantação ineficiente por parte dos gestores; (c) atrasos no feedback; e 
(d) excesso de ênfase em medidas financeiras. No que se refere à implantação ineficiente, muito se tem pesquisado sobre os procedimentos propriamente ditos, mas pouco sobre os pré-requisitos e as condições estruturais do sistema de controle gerencial para a sua implementação e uso.

Ittner e outros (2003) e Malina e Selto (2001) identificaram a existência de conflitos entre os gestores durante o processo de avaliação de desempenho em empresas que implantaram o BSC. Bessire e Baker (2005) criticam o que chamam de uma ênfase excessiva em uma metáfora mecânica. Para os autores, essa ênfase leva a um foco excessivo na implementação de uma técnica gerencial particular à custa de uma análise mais criteriosa sobre os propósitos da organização que deveria preceder a escolha e a implementação de um artefato do sistema de controle gerencial. Em outras palavras, as questões qualitativas da gestão não são priorizadas.

Prieto e outros (2006), em sua pesquisa que utiliza o estudo de múltiplos casos, analisaram 14 projetos de implementação de BSC e concluíram que alguns dos fatores críticos para o sucesso da implementação não foram praticados, dentre os quais destacaram: (a) falta de comprometimento da alta administração; (b) discussões pouco frequentes e não claras; (c) perspectivas não balanceadas; (d) BSC como um evento único e não como um processo contínuo; e (e) não definição dos papéis e responsabilidades.

\section{Atributos da contabilidade gerencial}

Os atributos da contabilidade gerencial são características qualitativas que as informações devem ter para atender objetivos específicos (MOORES; YUEN; 2001, p. 355). Esses atributos implicam a formalização de rotinas e procedimentos com grande uso de computadores, equipe técnica e modelagem financeira (MERCHANT, 1981, 1997; SIMONS, 1992, 1999).

O SICG deve ser analisado em duas dimensões: conteúdo e processo. A dimensão 'conteúdo' cobre os componentes técnicos (p. ex., sistemas de contabilidade por responsabilidade) e a forma da informação em termos de escopo, tempestividade, agregação e integração. A dimensão 'processo' trata de aspectos sociais e comportamentais associados com o desenho e a operação da dimensão conteúdo. A dimensão conteúdo, quanto ao escopo, tempestividade, agregação e integração (CHENHALL; MORRIS, 1986, p. 19) define a contabilidade em sentido amplo, que corresponde ao perfil mais rico quanto às características das informações. Tillema (2005, p. 106), por sua vez, percebendo que a dimensão conteúdo no sentido amplo não é a única possibilidade, propõe três dife- rentes classificações: amplo, estrito e médio. A dimensão conteúdo no sentido 'amplo', na perspectiva de Chenhall e Morris (1986), é aquela em que as informações estão voltadas para eventos atuais e futuros, e para os ambientes interno e externo, e, portanto, é a abordagem mais complexa e completa. A dimensão conteúdo no sentido escopo 'estrito' disponibiliza somente informações sobre eventos históricos. A dimensão conteúdo no sentido 'médio', embora disponibilize informações sobre eventos históricos e futuros, concentra-se no foco interno. Esta pesquisa considera o escopo médio (TILLEMA, 2005, p. 107), pois, ainda que trate de informação sobre eventos históricos (reais) e futuros, apenas a perspectiva interna da empresa foi considerada.

Moores e Yuen (2001), numa evolução do trabalho de Chenhall e Morris (1986), consolidaram o construto dos atributos do SICG em dois grupos: seleção da informação e apresentação. A 'seleção' inclui os elementos que correspondem às 'dimensões de conteúdo' da contabilidade e está relacionada com as características qualitativas da relevância e confiabilidade. Por sua vez, tendo em consideração que as organizações, dependendo de estratégias, estruturas ou estilos podem ser diferentes, a 'apresentação da informação' está relacionada com as características qualitativas da comparabilidade, abrangência e tempestividade e leva em conta alguns atributos, tais como nível de agregação, integração, escopo e tempestividade. $\mathrm{O}$ mapeamento ambiental, que, no estudo de Moores e Yuen (2001), compõe a dimensão conteúdo no sentido amplo, não foi utilizado na presente pesquisa, uma vez que ela foi estruturada no sentido médio, ou seja, considera apenas o foco interno, e, também, pelo fato de esse atributo ser bastante específico para empresas individuais.

Em seu estudo, Moores e Yuen (2001) não incluem a análise da característica qualitativa da materialidade, e incluem, na dimensão da 'apresentação da informação', a restrição da tempestividade, tal como já havia sido feito por Chenhall e Morris (1986). De acordo com Moores e Yuen (2001, p. 358), um SICG altamente formalizado disponibiliza uma variedade de artefatos a ser selecionada pelos gestores, desde relatórios financeiros tradicionais até sistemas sofisticados de contabilidade de custos, controle de qualidade e mapeamento ambiental. Sistemas sofisticados são aqueles que apresentam informações refletindo altos níveis de agregação e integração, amplo escopo e garantem relatórios tempestivos (CHENHALL; MORRIS, 1986). De maneira detalhada, as variáveis que compõem os atributos figuram no quadro a seguir.

Moores e Yuen (2001, p. 383) também mencionaram que o grupo referente à 'seleção' dos artefatos de conta- 
bilidade gerencial é muito mais importante do que o grupo que se refere à 'apresentação' de informações em vários níveis de formalidade do SICG em cada estágio do ciclo de vida. No estudo desses autores, os artefatos da dimensão 'seleção' foram analisados a partir de uma escala Likert de 7 pontos ( 1 = completamente não utilizada, 7 = usada em grande extensão), para identi- ficar a extensão em que os artefatos específicos eram usados. No presente estudo, o foco não foi o uso e sim a presença ou ausência de determinado artefato. Assim, no estudo de Moores e Yuen (2001), foi mensurado o "nível de uso", enquanto este estudo levantou se a empresa utiliza o artefato, mas não mensurou a intensidade desse uso.

\section{Quadro 1 - Atributos utilizados na pesquisa}

\begin{tabular}{|c|c|c|c|}
\hline \multicolumn{2}{|c|}{ SELEÇÃO } & \multicolumn{2}{|c|}{ APRESENTAÇÃO DA INFORMAÇÃO } \\
\hline $\begin{array}{l}\text { DEFINIÇÃO } \\
\text { CONCEITUAL }\end{array}$ & $\begin{array}{l}\text { MODUS OPERANDI } \\
\text { DA PESQUISA }\end{array}$ & $\begin{array}{l}\text { DEFINIÇÃO } \\
\text { CONCEITUAL }\end{array}$ & $\begin{array}{l}\text { MODUS OPERANDI } \\
\text { DA PESQUISA }\end{array}$ \\
\hline $\begin{array}{l}\text { 1. Demonstração de resulta- } \\
\text { dos mensais } \\
\text { 2. Balanço patrimonial } \\
\text { mensal } \\
\text { 3. Demonstração de fluxo } \\
\text { de caixa } \\
\text { 4. Contabilidade de custos } \\
\text { 5. Controle orçamentário }\end{array}$ & $\begin{array}{l}\text { 1. Demonstração de resultados } \\
\text { mensais } \\
\text { 2. Balanço patrimonial mensal } \\
\text { 3. Demonstração de fluxo de } \\
\text { caixa } \\
\text { 4. Tipo de método de custeio } \\
\text { e sistema de acumulação de } \\
\text { custo }\end{array}$ & $\begin{array}{l}\text { Agregação e integração: } \\
\text { a. Modelos de decisão } \\
\text { b. Combinação de dados ao } \\
\text { longo do tempo } \\
\text { c. Combinação de dados nas } \\
\text { áreas funcionais } \\
\text { d. Relatórios de interações en- } \\
\text { tre subunidades }\end{array}$ & $\begin{array}{l}\text { a. Modelos de decisão } \\
\text { b. Combinação de dados ao } \\
\text { longo do tempo } \\
\text { c. Combinação de dados nas } \\
\text { áreas funcionais } \\
\text { d. Relatórios de interações } \\
\text { entre subunidades }\end{array}$ \\
\hline $\begin{array}{l}\text { não participativo } \\
\text { 6. Controle orçamentário } \\
\text { participativo } \\
\text { 7. Orçamento de Capital } \\
\text { 8. Planejamento e previsões } \\
\text { de longo prazo } \\
\text { 9. Contabilidade por } \\
\text { responsabilidade } \\
\text { 10. Controle de qualidade }\end{array}$ & $\begin{array}{l}\text { 5. Processo de planejamento } \\
\text { estratégico, orçamento e con- } \\
\text { trole orçamentário centralizado } \\
\text { 6. Processo de planejamento } \\
\text { estratégico, orçamento e con- } \\
\text { trole orçamentário participativo } \\
\text { 7. Orçamento de capital } \\
\text { 8. Planejamento e previsões de } \\
\text { longo prazo } \\
\text { 9. Contabilidade por responsa- }\end{array}$ & $\begin{array}{l}\text { Escopo: } \\
\text { e. Interno } \\
\text { f. Externo } \\
\text { g. Financeiro } \\
\text { h. Não financeiro } \\
\text { i. Histórico } \\
\text { j. Futuro }\end{array}$ & $\begin{array}{l}\text { e. Interno } \\
\text { f. Externo } \\
\text { g. Financeiro } \\
\text { h. Não financeiro } \\
\text { i. Histórico } \\
\text { j. Futuro }\end{array}$ \\
\hline $\begin{array}{l}\text { 11. Mapeamento ambiental } \\
\text { 12. Informações finan- } \\
\text { ceiras para avaliação de } \\
\text { desempenho } \\
\text { 13. Critérios de longo } \\
\text { prazo para avaliação de } \\
\text { desempenho }\end{array}$ & $\begin{array}{l}\text { bilidade detalhada por centro } \\
\text { de custo, unidade de negócios, } \\
\text { etc. } \\
\text { 10. Programas de redução de } \\
\text { desperdício } \\
\text { 11. Não considerado neste } \\
\text { trabalho } \\
\text { 12. Informações financeiras } \\
\text { para avaliação de desempenho } \\
\text { 13. Critérios de longo prazo } \\
\text { para avaliação de desempenho }\end{array}$ & $\begin{array}{l}\text { Tempestividade: } \\
\text { k. Velocidade dos relatórios } \\
\text { I. Relatórios automatizados } \\
\text { m. Frequência dos relatórios } \\
\text { n. Time-lag no recebimento da } \\
\text { informação }\end{array}$ & $\begin{array}{l}\text { k. Velocidade dos relatórios } \\
\text { I. Integração dos ERP } \\
\text { m. Frequência dos relatórios } \\
\text { n. Time-lag no recebimento } \\
\text { da informação }\end{array}$ \\
\hline
\end{tabular}




\section{METODOLOGIA E DESIGN DA PESQUISA DE CAMPO}

Para a realização da presente pesquisa, optou-se por uma abordagem empírico-analítica de caráter quantitativo que investigou 106 empresas brasileiras, por meio de um levantamento (survey) que envolveu a aplicação de questionários estruturados com abordagem binária ( 0 = não e 1 = sim), para identificar o conhecimento e uso do BSC (variáveis de questionamento) e a estrutura de atributos da contabilidade gerencial (variáveis de perfil) presentes nas empresas da amostra. Para verificar a existência de associação entre as variáveis foi utilizado um teste não paramétrico (Kruskal Wallis), e a significância foi obtida em termos de presença ou ausência. Na primeira etapa do estudo, foram levantados fundamentos teóricos sobre (i) a utilidade dos artefatos do sistema de contabilidade gerencial para os gestores; (ii) o BSC, inclusive sobre dificuldades de implementação; e (iii) atributos da contabilidade gerencial.

A pesquisa caracteriza-se como exploratória, dado que visa a aprofundar a compreensão sobre o baixo nível de utilização do BSC nas empresas da amostra; e pode ser classificada como descritiva, uma vez que busca expor determinadas características das empresas da amostra e analisar as relações entre as variáveis pesquisadas. Com isso, os resultados servirão de base para construir hipóteses para estudos futuros.

O design da pesquisa fundamentou-se na proposta de Carmines e Zeller (1979) para a utilização de três elementos: critérios, conteúdo e estrutura para a validação dos achados. O sucesso da pesquisa depende de os três elementos estarem desenvolvidos de maneira equilibrada, embora, em muitos casos, isso não seja uma tarefa simples. A estrutura de elementos da pesquisa é baseada no racional desenvolvido por Henry (1990, p. 46), que liga 'natureza do estudo', 'definição da amostra', 'variáveis de interesse', 'subpopulações' e 'fontes das informações'.

A pesquisa foi desenvolvida utilizando-se de dados primários, coletados pelos autores, e foi estruturada para atingir os seguintes objetivos específicos:

- Identificar e apresentar os atributos da contabilidade gerencial.

- Identificar a existência do BSC na amostra definida.

- Verificar se existe alguma associação entre a existência do BSC e o perfil de atributos utilizados pelas entidades.

- No caso da existência do BSC, associar os atributos utilizados com os possíveis status de sua utilização, discriminados pelas variáveis de questionamento.

- Nesse sentido, os elementos considerados na estruturação da pesquisa foram os seguintes: definições dos estratos da população e da amostra, variáveis consideradas na pesquisa, testes aplicados sobre a amostra e coleta de dados.

\section{Definições dos estratos da população e da amostra}

A fim de responder à questão de pesquisa, a coleta de dados ocorreu a partir da segmentação da população originariamente considerada com 24 estratos agrupados em sete estratos: (1) comércio, (2) indústrias, (3) instituições financeiras, (4) telecomunicações, (5) serviços públicos, (6) serviços e (7) outros setores. Os passos seguintes foram:

- Estratificação da população, o que foi feito considerando duas dimensões: setores da economia e faturamento anual.

- Seleção da fonte dos dados, sendo a base de dados da revista brasileira Melhores e Maiores (2001) considerada como fonte adequada para se obter o total da população, com detalhes pelos diferentes setores, levando em conta o porte do faturamento.

- Identificação da população, sendo 2.281 organizações identificadas com um faturamento mínimo de US\$ 18 milhões, gerando um faturamento total, para a população, de US\$ 502 bilhões no ano de 2001.

- Definição dos estratos, sendo o tamanho de cada estrato planejado de acordo com a abordagem probabilística, considerando a média de população finita, planejada para $10 \%$ de erro em relação à média, o que implicou uma amostra de 125 entidades planejadas. Ao final da realização da pesquisa de campo, foram considerados como válidos 106 questionários, com erro estatístico de $12,2 \%$ pelo parâmetro da média.

- Reposição de elementos da amostra, nos casos em que a entidade não aceitou responder ao questionário, o que ocorreu dentro do estrato, de acordo com o setor e porte do faturamento, a partir do mesmo critério randômico que permitiu a seleção inicial.

\section{Variáveis consideradas na pesquisa}

A mensuração é o processo de ligação entre conceitos abstratos e as indicações empíricas (CARMINES; ZELLER, 1979). Como consequência, as variáveis de interesse identificadas foram tratadas a partir da aplicação do questionário à amostra descrita. Para um melhor entendimento, tais variáveis de interesse foram separadas em dois grupos: 'variáveis de questionamento' e 'variáveis de perfil' da contabilidade gerencial.

Variáveis de questionamento

Dizem respeito ao que se pretende pesquisar sobre o BSC, ou seja, a sua utilização na empresa. Colhida numa 
abordagem binária $(0=$ não e $1=\operatorname{sim})$, foi estruturada da seguinte forma: (i) o BSC é conhecido e utilizado na empresa (grupo 1); (ii) o BSC é conhecido mas não utilizado na empresa (grupo 2); (iii) o BSC é conhecido e está em implantação na empresa (grupo 3); e (iv) o BSC não é conhecido na empresa (grupo 4).

Variáveis de perfil

Correspondem às variáveis apresentadas no Quadro 1, separadas por seleção, apresentação e escopo, dentro da abordagem binária $(0=$ não e $1=\operatorname{sim})$.

\section{Testes aplicados sobre a amostra}

Antes da utilização dos dados coletados, foi apurado o Alfa de Cronbach, coeficiente de credibilidade mais utilizado (HAIR JR. e outros, 1998). Obteve-se o percentual de $69,8 \%$ de coeficiente estandardizado, o que significa que $69,8 \%$ do universo de possível impacto, baseado no mesmo número de itens, concordariam em $69,8 \%$ das vezes. A magnitude do indicador é muito boa e permitiu prosseguir com a análise.

Com a análise do teste não paramétrico (KRUSKAL WALLIS), foi possível identificar os atributos associados dentro do nível de significância de $95 \%$.

\section{Coleta de dados}

O questionário foi construído a partir de elementos da pesquisa, características que poderiam afetar as questões, consistência entre questões, sequência e saltos, bem como alguns pontos de checagem, todos reforçados ou ajustados no pré-teste. Por meio dele foram coletados os dados exigidos pela pesquisa, e sua aplicação antecedeu as entrevistas realizadas visando a validar dados coletados via questionário e obter uma percepção mais aprofundada acerca das variáveis pesquisadas. Todos os questionários foram enviados por meio eletrônico. Posteriormente, foram realizadas as entrevistas, cobrindo cerca de 30\% dos respondentes. $\mathrm{O}$ trabalho de campo foi iniciado em abril de 2002 e só terminou em novembro do mesmo ano.

\section{ANÁLISE E DISCUSSÃO}

A análise de dados foi feita dentro do desdobramento das quatro variáveis de questionamento, que são: 'grupo l', o BSC é conhecido e utilizado na empresa; 'grupo 2', o BSC é conhecido mas não utilizado na empresa; 'grupo 3', o BSC é conhecido e está em implantação na empresa; e 'grupo 4', o BSC não é conhecido na empresa. As duas variáveis consideradas para distinguir a amostra são o setor e o por- te (pelo faturamento). Pode se perceber (Tabela 1) que o setor da indústria é preponderante, com $49 \%$ do total da amostra, exercendo influência sobre o resultado. Alguns setores apresentam concentração, como, por exemplo, a indústria nos grupos 1 e 2, e instituições financeiras nos grupos 1 e 4 .

No que se refere ao porte de faturamento, classificado em faixas de valores em uma escala crescente (Tabela 2), pode-se mencionar que, relativamente, as empresas de maior porte se apresentam distribuídas nos vários grupos, enquanto as de menor porte estão concentradas no grupo 4. Essa característica é um dos fatores explicativos da menor utilização do balanced scorecard.

Ao analisar a associação entre os atributos (descritos no Quadro 1) da contabilidade gerencial e os quatro grupos, aplicando-se o teste não paramétrico Kruskal Wallis, foi possível identificar algumas variáveis como dentro do intervalo de significância de 95\%, conforme descritos na Tabela 3.

A significância foi obtida em termos de presença e ausência. Assim, na Tabela 3, o percentual de significância apresentado como 'ausência' indica uma não associação entre o atributo e o grupo correspondente da variável de questionamento, e o percentual de significância apresentado como 'presença' indica o inverso.

\section{Grupo 1: o BSC é conhecido e utilizado na empresa}

Dentro do elenco de atributos identificados com as empresas que utilizam o BSC, um refere-se a atributos de 'seleção' e um de 'escopo'. O atributo de seleção identificado foi a disponibilidade de 'informações financeiras para avaliação de desempenho' e o atributo referente ao escopo foi 'informações com escopo futuro' (medido pela disponibilidade do orçamento). A existência do BSC é consistente com tais atributos, pois a entidade que decide implementar o BSC tem que dispor de instrumentos voltados para o futuro e para a medição de desempenho. Prieto e outros (2006), ao questionarem os problemas com implementação do BSC como um evento único e não como um processo contínuo, indicam que a consistência e o foco claros do que obter do BSC são fundamentais e requerem dado grau de maturidade que requer a estrutura de informações proporcionada pelos atributos. Os achados de Besire e Baker (2005) que recomendam uma preparação de implementação muito focada nas prioridades do controle gerencial reforçam a percepção de que a maturidade e o interesse prospectivo são fundamentais para a implementação não ser uma variável técnica simplesmente.

Assim, o BSC surge como uma evolução da utilização madura do planejamento, demandando ligação e alinha- 
Tabela 1 - Distribuição das empresas por grupo e por setor da economia

\begin{tabular}{|c|c|c|c|c|c|c|}
\hline SETOR & GRUPO 1 & GRUPO 2 & GRUPO 3 & GRUPO 4 & TOTAL & $\%$ S TOTAL \\
\hline Setor 1 - Atacado, varejo e com.exterior & 1 & 9 & 1 & 2 & 13 & $12 \%$ \\
\hline Setor 2 - Indústrias - todas & 10 & 28 & 6 & 8 & 52 & $49 \%$ \\
\hline Setor 3 - Instituições financeiras & 4 & 6 & - & 4 & 14 & $13 \%$ \\
\hline Setor 4 - Telecomunicações & - & 3 & - & - & 3 & $3 \%$ \\
\hline Setor 5 - Serviços públicos & 1 & 6 & 1 & 1 & 9 & $8 \%$ \\
\hline Setor 6 - Serviços & 2 & 10 & 1 & 1 & 14 & $13 \%$ \\
\hline Setor 7 - Outros setores & - & 1 & - & - & 1 & $1 \%$ \\
\hline Total & 18 & 63 & 9 & 16 & 106 & $100 \%$ \\
\hline \multicolumn{7}{|l|}{ Em \% de participação } \\
\hline Setor 1 - Atacado, varejo e com.exterior & $6 \%$ & $14 \%$ & $11 \%$ & $13 \%$ & $12 \%$ & \\
\hline Setor 2 - Indústrias - todas & $56 \%$ & $44 \%$ & $67 \%$ & $50 \%$ & $49 \%$ & \\
\hline Setor 3 - Instituições financeiras & $22 \%$ & $10 \%$ & $0 \%$ & $25 \%$ & $13 \%$ & \\
\hline Setor 4 - Telecomunicações & $0 \%$ & $5 \%$ & $0 \%$ & $0 \%$ & $3 \%$ & \\
\hline Setor 5 - Serviços públicos & $6 \%$ & $10 \%$ & $11 \%$ & $6 \%$ & $8 \%$ & \\
\hline Setor 6 - Serviços & $11 \%$ & $16 \%$ & $11 \%$ & $6 \%$ & $13 \%$ & \\
\hline Setor 7 - Outros setores & $0 \%$ & $2 \%$ & $0 \%$ & $0 \%$ & $1 \%$ & \\
\hline Total & $100 \%$ & $100 \%$ & $100 \%$ & $100 \%$ & $100 \%$ & \\
\hline
\end{tabular}

Tabela 2 - Distribuição das empresas por grupo e porte de faturamento

\begin{tabular}{|c|c|c|c|c|c|c|}
\hline FATURAMENTO EM US\$ & GRUPO 1 & GRUPO 2 & GRUPO 3 & GRUPO 4 & TOTAL & $\%$ S TOTAL \\
\hline Até 50 milhões & 1 & 8 & - & 8 & 17 & $16 \%$ \\
\hline$>50<100$ & - & 11 & - & 2 & 13 & $12 \%$ \\
\hline$>100<250$ & 3 & 11 & 1 & 1 & 16 & $15 \%$ \\
\hline$>250<500$ & 4 & 7 & 2 & 1 & 14 & $13 \%$ \\
\hline$>500<1,000$ & 5 & 10 & 3 & 2 & 20 & $19 \%$ \\
\hline$>1,000<3,000$ & 3 & 15 & 2 & 2 & 22 & $21 \%$ \\
\hline$>3,000<30,000$ & 2 & 1 & 1 & - & 4 & $4 \%$ \\
\hline Total & 18 & 63 & 9 & 16 & 106 & $100 \%$ \\
\hline \multicolumn{7}{|l|}{ Em \% de participação } \\
\hline Até 50 milhões & $6 \%$ & $13 \%$ & $0 \%$ & $50 \%$ & $16 \%$ & \\
\hline$>50<100$ & $0 \%$ & $17 \%$ & $0 \%$ & $13 \%$ & $12 \%$ & \\
\hline$>100<250$ & $17 \%$ & $17 \%$ & $11 \%$ & $6 \%$ & $15 \%$ & \\
\hline$>250<500$ & $22 \%$ & $11 \%$ & $22 \%$ & $6 \%$ & $13 \%$ & \\
\hline$>500<1,000$ & $28 \%$ & $16 \%$ & $33 \%$ & $13 \%$ & $19 \%$ & \\
\hline$>1,000<3,000$ & $17 \%$ & $24 \%$ & $22 \%$ & $13 \%$ & $21 \%$ & \\
\hline$>3,000<30,000$ & $11 \%$ & $2 \%$ & $11 \%$ & $0 \%$ & $4 \%$ & \\
\hline Total & $100 \%$ & $100 \%$ & $100 \%$ & $100 \%$ & $100 \%$ & \\
\hline
\end{tabular}


mento entre estratégico e tático. Sem esses atributos, os benefícios potenciais do BSC não podem ser plenamente alcançados. Paranjape e outros (2006) constataram, dentre as causas do insucesso, a implantação ineficiente por parte dos gestores e atrasos no feedback. O atraso pode estar ligado a buscar o BSC ser ter estrutura de informações que permita a sua operacionalização, inclusive para que o feedback ocorra de maneira constante, oportuna e eficaz. A disponibilidade dos atributos indicados proporciona entendimento de que, na amostra considerada, as condições necessárias estão disponíveis.

Rosário (2005, p. 316) observa que os resultados de seu estudo acerca do forte impacto do processo de revisão do diagrama causal/mapa estratégico na aprendizagem confirmam que o processo de feedback associado ao ciclo de modelação e revisão dos pressupostos dos gestores acerca das relações causa-efeito conduzem a uma melhor compreensão do contexto empresarial, que, por sua vez, induzem uma melhor performance de gestão.

\section{Grupo 2: o BSC é conhecido mas não utilizado na empresa}

O grupo de empresas que conhece o BSC enquanto conceito mas não o utiliza corresponde ao maior de todos, com 63 empresas. Existem dois atributos importantes, ou seja, que se enquadram no nível de significância estabelecido: um deles referente à 'seleção' da informação ('ter conta- bilidade de custos') e um referente à 'apresentação das informações' (ter 'informações não financeiras'). O atributo 'ter contabilidade de custos' pode se relacionar com o perfil da amostra, ou seja, pelo fato de ser preponderante na indústria, que corresponde à maior parte da amostra.

O fato de ter sistema de custos e informações não financeiras indica que esse agrupamento tem razoável nível de estrutura para o gerenciamento de suas atividades. O que levaria uma organização que conheça o BSC a adotálo? Alguma demanda para planejamento estruturado. O comentário de Scapens (1994) sobre a pouca utilização de artefatos ainda se faz presente, mas ele se preocupou mais em estudar o que e como as organizações usam, não privilegiando o estudo dos porquês. A contribuição identificada no grupo 1 é relevante no sentido de que aquilo que foi encontrado no grupo que usa o BSC é algo que não foi encontrado nos demais. Trata-se do antecedente mais relevante, ou seja, de estar a organização claramente impregnada pela necessidade de ter um processo estruturado de planejamento. Pensar no BSC sem essa estrutura não leva a uma visão consistente e madura do artefato.

\section{Grupo 3: o BSC é conhecido e está em implantação na empresa}

Nenhum dos atributos foi associado, pelo teste de Kruskal Wallis, ao nível de 95\% de significância, ao grupo das empresas que conhecem o conceito e que estão implantando

Tabela 3 - Atributos associados aos grupos de utilizaçãodo BSC

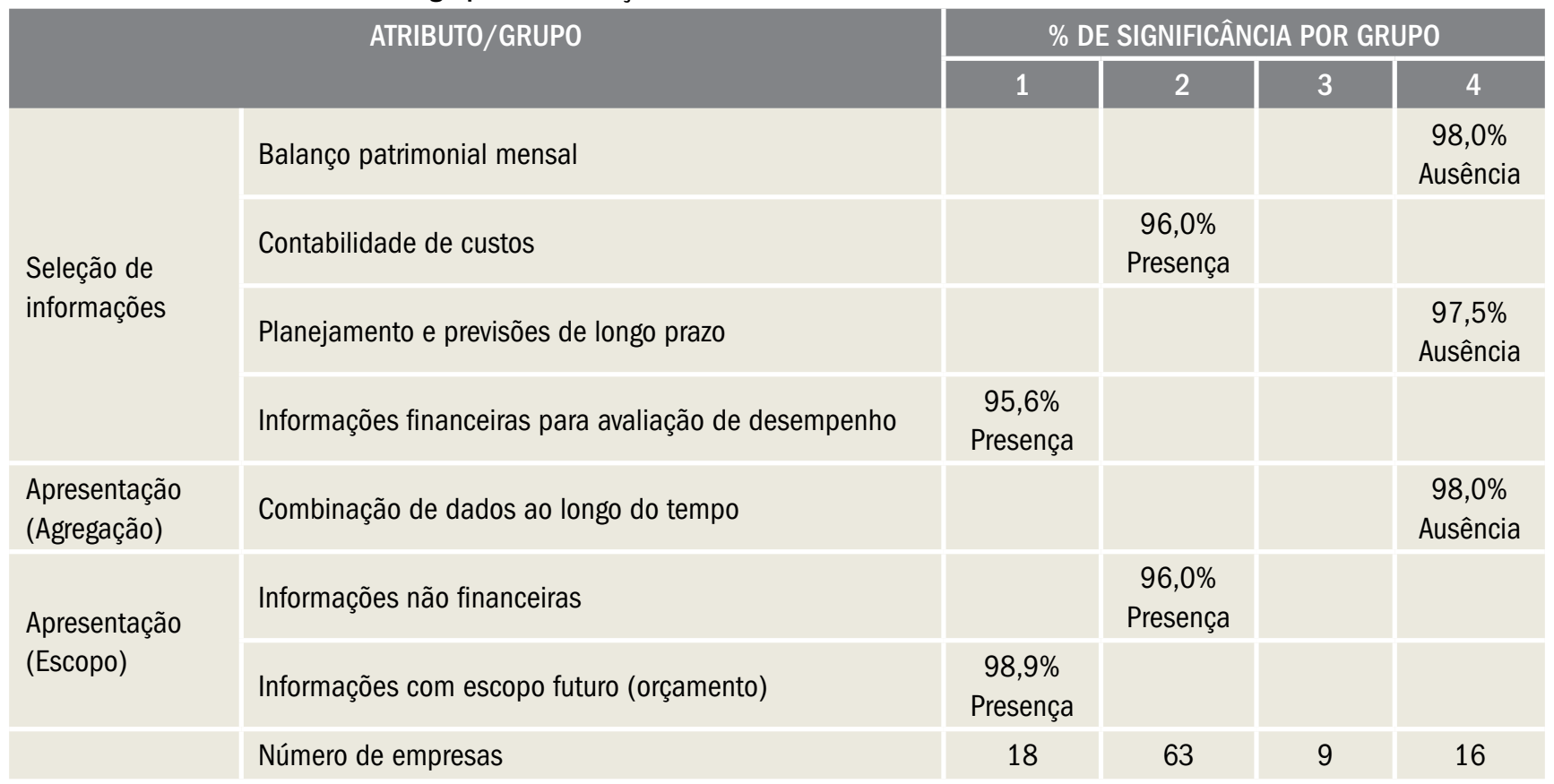


o BSC na empresa. Isso pode ter ocorrido em função do pequeno número de empresas que compõe o grupo (somente 9 empresas).

\section{Grupo 4: o BSC não é conhecido na empresa}

Conforme se pode observar, no grupo de empresas que não conhecem e, portanto, não 'utilizam' o BSC como parte de suas ferramentas gerenciais, houve ausência de dois atributos da 'seleção de informações' (balanço patrimonial mensal, e planejamento e previsões de longo prazo) e um de 'apresentação' (combinação de dados ao longo do tempo). Demonstrações contábeis mensais constituem um mínimo esperado para se poder gerenciar uma entidade, $e$ isso não foi encontrado nessas entidades. Adicionalmente, um processo de planejamento de longo prazo é algo fundamental para o desenvolvimento dos negócios. Sem isso, pode-se comprometer a implementação e o alcance dos benefícios potenciais do BSC. Já foi demonstrada, para o mesmo ambiente, a dependência de uma contabilidade de bom nível para que se possa dispor de um processo de planejamento estruturado.

O entendimento de Chenhall (2003, p. 132), que considera que a percepção de utilidade para os gestores seja fundamental para a sua adoção, é relevante nessa análise. Por sua vez, esse entendimento depende da busca de soluções para problemas de gestão. O desconhecimento da existência da ferramenta pode ser explicado pelo fato de que o BSC pode ser usado para varias funções na empresa, podendo sua implementação ser conduzida por diferentes áreas, o que torna difusa a sua demanda. Levando em conta que as empresas deste grupo são predominantemente de menor porte, é provável que os problemas mais relevantes dessas organizações não demandem um artefato tão complexo como o BSC. A ausência de uma preocupação prospectiva indica esse estagio. O BSC, que é uma ferramenta relativamente sofisticada e complexa em termos de envolver a organização como um todo, não pode prosperar num ambiente em que atributos básicos não estejam presentes, sejam eles ligados à estrutura do sistema de informações ou mesmo aos recursos humanos envolvidos na sua utilização.

\section{CONCLUSÕES}

Os objetivos (gerais e específicos) da pesquisa foram alcançados na medida em que os resultados permitiram constatar a existência, para a amostra considerada, de associação entre o perfil de atributos da contabilidade gerencial (abordagem proposta por Moores e Yuen) e a utilização do BSC nas empresas, o que responde à questão de pesquisa formulada. Os atributos da contabilidade gerencial constituem a base de sustentação da estrutura de um sistema de controle gerencial para que a entidade possa dispor de ferramentas gerenciais. Constatou-se que a grande maioria das empresas da amostra (59\%) conhece o BSC, mas não usa (grupo 2). Do total de empresas da amostra, 26\% conhecem o BSC (17\% utilizam e 9\% estão em fase de implementação) e 15\% não conhecem e, portanto, não utilizam o BSC.

Os resultados revelam que alguns dos atributos, tanto da seleção como da apresentação das informações, foram identificados nas empresas que 'conhecem e utilizam o BSC' (disponibilidade de informações financeiras para avaliação de desempenho e informações com escopo futuro), naquelas que 'o conhecem mas não o utilizam' (presença da contabilidade de custos e informações não financeiras) e naquelas que 'não o conhecem nem o utilizam' (ausências do balanço patrimonial mensal, planejamento e previsões de longo prazo, e combinação de dados ao longo do tempo). Os atributos presentes, por um lado, e os atributos ausentes, por outro, permitem aos gestores entender e direcionar esforços para compreender melhor as causas de não implementação e, até mesmo, de fracassos no tratamento de ferramentas gerenciais.

A principal contribuição do trabalho para o campo do conhecimento da administração reside no potencial de diagnóstico de requisitos mínimos a serem atendidos para suportar o BSC como mecanismo de feedback capaz de influenciar positivamente a aprendizagem dos gestores e a qualidade da gestão. A utilidade desse conhecimento está na orientação aos gestores, que, muitas vezes, identificam as ferramentas que desejam dispor nas instituições, mas não necessariamente percebem os pré-requisitos para que sejam efetivas. Particularmente, os atributos ligados relacionados ao processo de planejamento, indicando que o BSC é o prolongamento de um processo que se inicia com a demanda por projeções de resultados.

Visando sugerir a realização de novas pesquisas e sem a pretensão de esgotar as possibilidades, algumas possíveis explicações adicionais podem ser consideradas:

- Artefatos similares ou derivados do BSC podem estar sendo utilizados com outros nomes e formas e não foram captados pela pesquisa;

- O BSC é utilizado em determinada área, mas não em toda a empresa, o que limita a presente pesquisa, pois uma resposta binária, do tipo "sim" ou "não", pode não permitir tal ocorrência; e

- A pesquisa reflete o estágio de desenvolvimento da área no país. 
Analisando a primeira alternativa, provavelmente ela deve contribuir para uma parte da explicação, mas isso exigirá a realização de uma nova pesquisa de campo. $\mathrm{O}$ mesmo ocorre com a segunda alternativa, muito embora tenha ficado claro que algumas organizações utilizam o BSC em algumas áreas funcionais (como Marketing, por exemplo), mas não na empresa como um todo. Em síntese, sabe-se que ela ocorre, contudo não é possível avaliar a sua magnitude sem nova pesquisa. Da mesma forma, pode se perceber que algumas empresas utilizam o BSC em uma unidade de negócios, porém não em todas. Esse tipo de ocorrência torna a questão do perfil mais complicada, já que a questão foi aplicada de forma binária, como destacado anteriormente. A terceira alternativa, por sua vez, corresponde a uma conclusão que dependeria de variáveis ainda não apresentadas $\mathrm{e}$, analogamente às alternativas anteriores, exige uma nova pesquisa.

\section{NOTA DE AGRADECIMENTO}

Os autores agradecem a FAPESP, o CNPq e a FIPECAFI pelo apoio proporcionado ao projeto-eixo que originou este trabalho.

\section{REFERÊNCIAS}

ABERNETHY, M. A; VAGNONI, E. Power, organization design and managerial behaviour. Accounting, Organizations and Society, v. 29, n. 3-4, p. 207-225, 2004.

AX, C; BJORNENAK, T. Bundling and diffusion of management accounting innovations: the case of balanced scorecard in Sweden. Management Accounting Research, v. 16, n. 1, p. 1-20, 2005.

BESSIRE, D; BAKER, C. R. The French tableau de bord and the American balanced scorecard: a critical analysis. Critical Perspectives on Accounting, v. 16, n. 6 , p. $645-664,2005$.

BURNS, J; SCAPENS, R. W. Conceptualizing management accounting change: an institutional framework. Management Accounting Research, v. 11, n. 1, p. 3-25, 2000.

CARMINES, E. G; ZELLER, R. A. Reliability and validity assessment. London: Sage, 1979

CHENHALL, R. H. Management control systems design within its organizational context: findings from contingency-based research and directions for the future. Accounting, Organizations and Society, vol. 28, n. 2-3, p. $127-168,2003$
CHENHALL, R. H. Integrative strategic performance measurement systems, strategic alignment of manufacturing, learning and strategic outcomes: an exploratory study. Accounting, Organization and Society, v. 30 n. 5, p. 395-422, 2005.

CHENHALL, R. H; MORRIS, D. The impact of structure, environment, and interdependence on the perceived usefulness of management accounting systems. The Accounting Review, v. 61, n. 1, p. 16-35, January 1986.

CHONG, V. K. Management accounting systems, task uncertainty and managerial performance: a research note. Accounting Organization and Society, v. 21, n. 5, p. 415-421, 1996.

DAVIS, S; ALBRIGHT, T. An investigation of the effect of balanced scorecard implementation on financial performance. Management Accounting Research, v. 15, n. 2, p. 135-153, 2004.

EPSTEIN, M; MANZONI, J. F. Implementing corporate strategy: from tableaux de bord to balanced scorecards. European Management Journal, v. 16, n. 2, p. 190-203, 1998.

FERNANDES, K. J; RAJA, V; WHALLEY, A. Lessons from implementing the balanced scorecard in a small and medium size manufacturing organization. Technovation, v. 26, n. 5-6, p. 623-634, 2006.

FREZATTI, F. Management accounting profile of firms located in Brazil: a field study. Brazilian Administrative Review, v. 3, n. 1, p. 73-87, 2005.

HAIR JR. J; ANDERSON, R. E; TATHAM, R. L; BLACK, W. C. Multivariate Data Analysis. 5th ed. Engleswood Cliffs, NJ: Prentice-Hall, 1998.

HENRY, G. Practical Sampling. London: Sage, 1990

ITTNER, C; LARCKER, D; MEYER, M. Subjectivity and the weighting of performance measures: evidence from a balanced scorecard. The Accounting Review, v. 3, n. 78, p. 725-758, 2003

KALD, M; NILSSON, F. Performance measurement at Nordic companies European Management Journal, v. 18, n. 1, p. 113-127, 2000.

KAPLAN, R. S; NORTON, D. P. Balanced Scorecard: Translating Strategy into Action. Boston: Harvard Business School Press, 1996.

KAPLAN, R. S; NORTON, D. P. The balanced scorecard: measures that drive performance. Harvard Business Review, v. 70, n. 1, p. 71-79, 1992

KAPLAN, R. S; Norton, D. P. Organização orientada para a estratégia: como as empresas que adotaram o balanced scorecard prosperaram no novo ambiente de negócios. 5. ed. Rio de Janeiro: Campus, 2001 


\section{ARTIGOS - BSC E A ESTRUTURA DE ATRIBUTOS DA CONTABILIDADE GERENCIAL: UMA ANÁLISE NO AMBIENTE BRASILEIRO}

MALINA, M. A.; SELTO, F. H. Communicating and controlling strategy: an empirical study of the effectiveness of the balanced scorecard. Journal of Management Accounting Research, v. 13, n. 1, p. 47-90, 2001.

MERCHANT, K. A. The design of the corporate budgeting system: influences on managerial behavior and performance. The Accounting Review, v. 56, n. 4, p. 813-829, 1981.

MOORES, K; YUEN, S. Management accounting systems and organizational configuration: a life-cycle perspective. Accounting, Organizations and Society, v. 26, n. 4-5, p. 351-389, 2001

NEELY, A; AUSTIN, R. Measuring operations performance: past, present and future. In: Performance Measurement, Conference. Proceedings... Cambridge, MA, p. 419-426, 2000.

NORREKLIT, H. The balanced scorecard: what is the score? A rhetorical analysis of the balanced scorecard. Accounting, Organizations and Society, v. 28, n. 6 , p. $591-619,2003$

PARANJAPE, B; ROSSITER, M; PANTANO, V. Insights from the balanced scorecard performance measurement systems: successes, failures and future - a review. Measuring Business Excellence, v. 10, n. 3, p. 4-14, 2006.

PIERCE, B; O'DEA, T. Management accounting information and the needs of managers: perceptions of managers and accountants compared. The British Accounting Review, v. 3, n. 35, p. 257-290, 2003.

PRIETO, V. C; PEREIRA, F. L. A; CARVALHO, M. M. de; LAURINDO, F. J. B. Fatores críticos na implementação do balanced scorecard. Gestão e Produção, São Carlos, v. 13, n. 1, p. 81-92, 2006

RODRIGUES, M. C. P. Marco lógico e BSC: um mesmo método e uma velha ideia? In: ENCONTRO NACIONAL DA ASSOCIAÇÃO NACIONAL DOS PROGRAMAS DE PÓS-GRADUAÇÃO EM ADMINISTRAÇÃO, 25, 2001, Campinas. Anais. Rio de Janeiro: ANPAD, 2001.

ROSA, A. X. Tableau de bord de gestion. In: ENCONTRO NACIONAL DA ASSOCIAÇÃO NACIONAL DOS PROGRAMAS DE PÓS-GRADUAÇÃO EM ADMINISTRAÇÃO, 24, 2000, Florianópolis. Anais. Florianópolis: ANPAD, 2000
ROSÁRIO, C. E. C. R. do. Análise da eficácia do balanced scorecard e o efeito da (des)semelhança estrutural do modelo mental na decisão estratégica: uma abordagem baseada em simulação. Tese de Doutorado, Instituto Superior de Ciências do Trabalho e da Empresa, Lisboa, 2008.

SCAPENS, R. Never mind the gap: towards an institutional perspective in management accounting practice, Management Accounting Review, v. 5, n. 3-4, p. 301-321, 1994.

SOARES JÚNIOR, H. Experiências de implementação do BSC: como as empresas estão implantando o BSC no Brasil. Rio de Janeiro, 2003. Dissertação de Mestrado, Faculdade de Administração e Ciências Contábeis da Universidade Federal do Rio de Janeiro, Rio de Janeiro, 2003.

SPECKBACHER, G; BISCHOF, J; PFEIFFER, T. A descriptive analysis on the implementation of balanced scorecards in German-speaking countries. Management Accounting Research, v. 14, n. 4, p. 361-387, 2003.

TILLEMA, S. Towards an integrated contingency framework for MAS sophistication - case study on the scope of accounting instruments in Dutch power and gas companies. Management Accounting Research, v. 16, n. 1, p. 101-129, 2005

TONCHIA, S. Linking performance measurement system to strategic and organizational choices. International Journal of Business Performance, v. 2, n. $1-3$, p. $15-29,2000$

WATTS, R; ZIMMNERMAN, J. Positive Accounting Theory. Englewood Clifs, NJ: Prentice-Hall, 1986

WONG-ON-WING, B; GUO, L; LI, W; YANG, D. Reducing conflict in balanced scorecard evaluations. Accounting, Organizations and Society, v. 32, n. 4-5, p. 363-377, 2007.

WOULTERS, M; VERDAASDONK, P. Supporting management decisions with ex ante accounting information. European Management Journal, v. 1 , n. 20 , p. $62-84,2002$ 